\title{
Path Following for Mobile Manipulators
}

\author{
Rajan Gill Dana Kulić Christopher Nielsen
}

\begin{abstract}
This paper presents a framework of path following via set stabilization for mobile manipulator systems. The mobile manipulator is modelled as a single redundant dynamic system. The mobile base considered belongs to a large class of wheeled ground vehicles, including those with nonholonomic constraints. Kinematic redundancies are resolved by designing a controller that solves a suitably defined constrained quadratic optimization problem, which can be easily tuned by the designer to achieve various desired poses. By employing partial feedback linearization, the proposed path following controller has a clear physical meaning. The desired path to be followed is a spline in the output space of the system. The controller simultaneously controls the manipulator and mobile base. The result is a unified path following controller without any trajectory planning performed on the mobile base. The approach is experimentally verified on a 4-degree-of-freedom (4-DOF) manipulator mounted on a differential drive mobile platform.
\end{abstract}

\section{Introduction}

The problem of designing feedback control laws that make a robot follow a desired curve in its workspace can be broadly classified as either a trajectory tracking or path following problem. Trajectory tracking consists of tracking a curve with an assigned time parametrization [10]. This approach may not be suitable for certain applications as it may limit the attainable performance, be unnecessarily demanding or even infeasible [8]. On the other hand, path following or manoeuvre regulation controllers, as previously defined in [10], drive a system's output to a desired path and make the output traverse the path without a pre-specified time parametrization. In general, path following results in smoother convergence to the desired path com-

Department of Electrical and Computer Engineering, University of Waterloo, 200 University Avenue West, Waterloo, Ontario, Canada, N2L 3G1, e-mail: \{rjgill, dkulic, cnielsen\} @ uwaterloo.ca 
pared to trajectory tracking, the control signals are less likely to saturate [17], and performance limitations for non-minimum phase systems are removed [1]. Path following controllers can also render the desired path attractive and invariant [11]. This is a useful property in robotics, since if a disturbance or obstacle is preventing the output of a robot to proceed along the path, the robot will stay on the path until the disturbance is removed [11]. Furthermore, if the robot's output is perturbed so that it leaves the path, the path following controller will drive the output back to the desired path. There are several approaches for path following, see [9] for a complete literature review.

The majority of applications and experiments of mobile manipulators do not simultaneously control the manipulator and the mobile base. That is, they control the manipulator and the mobile base in a sequential, not parallel (as is done in this paper), manner [21, 18, 3, 25]. Mobile manipulator systems can also be modelled as a single redundant system [26]. Previous works have tackled the trajectory tracking problem by resolving the redundancies at the kinematic level, but, unlike in this paper, a trajectory for the end-effector as well as the mobile base is specified [22, 12]. In this paper, the motion planning process is simplified since there is no need to plan separate trajectories (or paths) for the base and the end-effector. Khatib extended the dynamic-level redundancy resolution developed for torque-input model of robot manipulators [14] to holonomic mobile platforms [15], whereas the approach in this paper can also be applied directly to combined motor-input manipulator mounted on nonholonomic ground vehicles. In [26], the authors look at the coordinated mobile manipulator problem, where, unlike in this paper, the manipulator dynamics are neglected, to track a reference trajectory in the workspace of the mobile platform while maximizing the manipulability measure.

In this paper, we apply the path following control approach of [9] to general mobile manipulator systems. We use dynamic extension [2,13] to transform the dynamics of the mobile manipulator system that are tangential and transversal to the path into linear subsystems, and doesn't result in a singularity when the base velocity is zero unlike in previous work [2]. The remaining dynamics, redundant to following the path, appear as internal dynamics of the closed-loop system. A novel redundancy resolution technique is used at the dynamic level, and is experimentally shown to yield bounded internal dynamics, while maintaining a preferred manipulator posture. This scheme can easily be tuned by the designer to achieve various desired poses. The result is an automatic unified path following controller that compensates for the dynamics of the mobile manipulator system, controlling the mobile base and manipulator simultaneously, rendering a desired path in the output space of the system to be attractive and invariant. There is no trajectory that needs to be planned and tracked by the manipulator nor the mobile base, and the coordination between the two is done automatically by our proposed path following controller.

Notation: Let $\langle x, y\rangle$ denote the inner product of vectors $x$ and $y$ in $\mathbb{R}^{n}$. The Euclidean norm of a vector and induced matrix norm are both denoted by $\|\cdot\|$. The notation $s \circ h: A \rightarrow C$ represents the composition of maps $s: B \rightarrow C$ and $h: A \rightarrow B$. The $i$ th element of a vector $x$ is denoted $x_{i}$, and the row $i$ to $j$ and column $k$ to $l$ submatrix of $\mathrm{A}$ is denoted as $A_{i: j, k: l}$. The symbol $:=$ means equal by definition. 
Given a $C^{1}$ mapping $\phi: \mathbb{R}^{n} \rightarrow \mathbb{R}^{m}$ let $\mathrm{d} \phi_{x}$ be its Jacobian evaluated at $x \in \mathbb{R}^{n}$. If $f, g: \mathbb{R}^{n} \rightarrow \mathbb{R}^{n}$ are smooth vector fields we use the following standard notation for iterated Lie derivatives $L_{f}^{0} \phi:=\phi, L_{f}^{k} \phi:=L_{f}\left(L_{f}^{k-1} \phi\right)=\left\langle\mathrm{d} L_{f}^{k-1} \phi_{x}, f(x)\right\rangle$, $L_{g} L_{f} \phi:=L_{g}\left(L_{f} \phi\right)=\left\langle\mathrm{d} L_{f} \phi_{x}, g(x)\right\rangle$. Finally, $0_{n \times m}$ is the $n$ by $m$ zero matrix and $I_{n \times m}$ is a $n$ by $m$ matrix of zeros with ones on its main diagonal.

\section{Class of Systems}

We consider a fully actuated manipulator mounted on a mobile ground vehicle. For simplicity, the combined systems are assumed to be decoupled. This is a valid system model assumption for general mobile manipulator systems [4, 15] and for our experimental platform (Sec. 5).

Manipulator Subsystem: The first subysystem is a fully actuated manipulator system with $N$ configuration variables and $N$ control inputs. The dynamic model is of the familiar form [23]

$$
M(q) \ddot{q}+C(q, \dot{q}) \dot{q}+G(q)=A(q) u_{m}
$$

where $u_{m} \in \mathbb{R}^{N}$ is the manipulator control input (usually motor voltages [23]), and $(q, \dot{q}) \in \mathbb{R}^{N} \times \mathbb{R}^{N}$ are joint positions and velocities ${ }^{1}$.

The output of system (1), the task space of the manipulator, lives in a $P$ dimensional space, and is given in local frame $\mathscr{B}$ attached to the vehicle base (see Fig. 1) as

$$
y_{m}=h(q)
$$

where $h: \mathbb{R}^{N} \rightarrow \mathbb{R}^{P}$ is smooth. In addition, let $J: \mathbb{R}^{N} \rightarrow \mathbb{R}^{P \times N}, q \mapsto \mathrm{d} h_{q}$ represent the manipulator Jacobian [23]. We assume, without loss of generality, that $P \geq 3$ and that the first 3 components of $h$ correspond to the Euclidean position of the endeffector in the local frame $\mathscr{B}$ (Fig. 1). Any additional outputs $(P>3)$ of $h$ represent orientations of the end-effector in the world frame. For example, if $P=4$, the fourth row of $h$ could represent the end-effector angle with respect to the horizontal ground plane [20].

Vehicle Subsystem: The position $x^{b} \in \mathbb{R}^{2}$ and orientation $\theta \in \mathbb{R}$ of frame $\mathscr{B}$ in an inertial frame $\mathscr{O}$ (see Fig. 1) are governed by the vehicle's dynamics. A general vehicle kinematic model is given as [5]:

$$
\begin{aligned}
{\left[\begin{array}{c}
\dot{x}^{b} \\
\dot{\theta}
\end{array}\right] } & =R(\theta) \Sigma(\sigma) \gamma_{b} \\
\dot{\sigma} & =\gamma_{s}
\end{aligned}
$$

\footnotetext{
${ }^{1}$ The results of this paper do not rely on the assumption that the state space be Euclidean. One could replace $\mathbb{R}^{N}$ by a smooth Riemannian manifold. Nonetheless, we assume $x \in \mathbb{R}^{N}$ to avoid unnecessarily cumbersome notation.
} 
where $R(\theta) \in \mathrm{SO}(3)$ is the rotation matrix

$$
R(\theta):=\left[\begin{array}{ccc}
\cos (\theta) & -\sin (\theta) & 0 \\
\sin (\theta) & \cos (\theta) & 0 \\
0 & 0 & 1
\end{array}\right]
$$

$\Sigma: \mathbb{R}^{\delta_{s}} \rightarrow \mathbb{R}^{3 \times \delta_{b}}$ is a full rank matrix [5, pg. 283] that depends on the steering angle $\sigma \in \mathbb{R}^{\delta_{s}}$ of the steering wheels, $\delta_{s} \in\{0,1,2\}$ is the degree of steerability [5, pg. 273], $\delta_{b} \in\{1,2,3\}$ is the degree of mobility of the mobile base [5, pg. 272], and $\gamma_{b} \in \mathbb{R}^{\delta_{b}}, \gamma_{s} \in \mathbb{R}^{\delta_{s}}$ are the inputs to the vehicle. The degrees of mobility and steerability $\delta_{b}, \delta_{s}$ depend on the design of the vehicle and satisfy the constraint $\delta_{b}+\delta_{s} \in\{2,3\}$. The kinematic model (3) encompasses many wheeled-ground vehicle designs including car-like vehicles, unicycles (see Sec. 5), and omnidirectional vehicles [5, Chapter 7.2]. If the vehicle has no steering wheels, such as those found on differential drive robots (see Sec. 5), then $\delta_{s}=0$ and $\Sigma$ is a constant matrix.

Output Model:
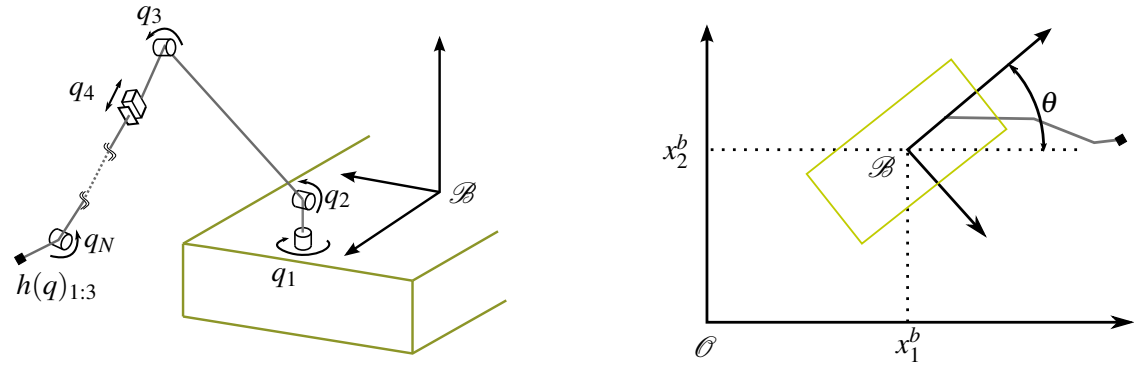

Fig. 1 Schematic of the mobile manipulator. The end-effector position $h(q)$ is expressed in the local-frame $\mathscr{M}$ attached to the bottom of the mobile base. The position and orientation of the local-frame in the inertial frame $\mathscr{O}$ are determined by $x^{b}, \theta$.

The $p$-dimensional output of the overall system is taken to be manipulator position $h(q)$ in the inertial frame in the following sense

$$
y:=H\left(q, x^{b}, \theta\right)=\left[\begin{array}{cc}
R(\theta)_{1: 2,1: 2} & 0_{2 \times(P-2)} \\
0_{(p-2) \times 2} & I_{(p-2) \times(P-2)}
\end{array}\right] h(q)+\left[\begin{array}{c}
x_{1}^{b} \\
x_{2}^{b} \\
0_{(p-2) \times 1}
\end{array}\right] .
$$

where $P \geq p \geq 2$ is the dimension of the output space. If $p=2$, then only the planar position of the end-effector in the inertial frame is of concern. If both $P>3$ and $p>3$, then a component of the end-effector orientation is also to be specified.

Assumption 2.1 (Dexterity) The mobile manipulator satisfies $N+\delta_{b} \geq p$ (see Remark 1), i.e., the system has enough degrees of freedom to follow arbitrary paths in the reachable space contained in $\mathbb{R}^{p}$. 


\section{Problem Formulation}

The problem studied in this paper is to find a continuous feedback control law for $u_{m}, \gamma_{b}, \gamma_{s}$ for the mobile manipulator system such that the output $y$ exponentially approaches a path in the output space $\mathbb{R}^{p}$ and traverses it towards a given desired position or with a given velocity profile. This will be done via set stabilization [19]. Since the mobile manipulator system is redundant, the redundancy is used to avoid manipulator joint limits and steering limits (if any) of the vehicle.

We assume that we are given a $(p+1)$-times continuously differentiable, parametrized curve in the output space of the mobile manipulator system,

$$
\sigma: \mathbb{R} \rightarrow \mathbb{R}^{p}
$$

which can be generated by spline interpolating a series of waypoints [6]. If $p>$ 3 , then the path also specifies a desired orientation of the end-effector. Given $\left[\lambda_{\min }, \lambda_{\max }\right] \subseteq \mathbb{R}$, the desired path is the set $\mathscr{P}:=\sigma\left(\left[\lambda_{\min }, \lambda_{\max }\right]\right)$. We assume that the curve (7) is framed:

Assumption 3.1 (framed curves) For all $\lambda \in\left[\lambda_{\min }, \lambda_{\max }\right], \sigma^{\prime}(\lambda), \ldots, \sigma^{(p)}(\lambda)$ are linearly independent.

Assumption 3.1 allows for the Frenet-Serret frame (FSF) of the path to be welldefined [16], and the prospect of it being violated is very low when spline-interpolating waypoints (see Sec. 5). The use of FSFs for control of mobile robots is a well-known technique [24, 9]. In this paper we use Assumption 3.1 to generate a zero-level set representation of $\mathscr{P}$ in the state space of (12). Assumption 3.1 can be relaxed if a zero-level set representation of the path is already available, see [9].

The goals of this paper are to determine a control law for $u_{m}, \gamma_{b}, \gamma_{s}$ such that

PF 1: The set $\mathscr{P}$ is rendered output invariant and locally attractive ${ }^{2}$.

PF 2: A desired position or velocity profile (tangent to the path) $\eta^{\text {ref }}: \mathbb{R}_{\geq 0} \rightarrow \mathbb{R}$ is tracked.

PF 3: Redundant dynamics (internal dynamics for the closed-loop system) remain bounded while ensuring joint limits of the manipulator are respected.

\section{Path Following Control Design}

The control design approach relies on the output $y$ having a well-defined relative degree of $\{2, \ldots, 2\}$. That is, the control inputs $u_{m}, \gamma_{b}, \gamma_{s}$ all appear in the second time-derivative of $y$. It can be shown that this is not the case: the control inputs $\gamma_{b}$ appear too soon by one derivative. Hence, by delaying the appearance of this input

\footnotetext{
${ }^{2}$ Invariance: if for some time $t=0$ the state $x(0)$ is appropriately initialized with $y=H(x(0)) \in \mathscr{P}$, then $(\forall t \geq 0) H(x(t)) \in \mathscr{P}$. Attractiveness: for initial conditions $x(0)$ such that the output $H(x(0))$ is in a neighbourhood of the desired path $\mathscr{P}, H(x(t)) \rightarrow \mathscr{P}$ as $t \rightarrow \infty$.
} 
via dynamic extension, we can make $y$ have the desired relative degree. Then a "virtual" output is constructed that incorporates the desired path (7). Through partial feedback linearization, the virtual output partitions the system dynamics into controllable transversal and tangential sub-systems that govern the motion transversal to the path and along the path, respectively, and a redundant sub-system that governs the motion of the system that does not produce any output motion. A novel redundancy resolution approach is then used to ensure the redundant dynamics remain bounded, while maintaining joint limits of the manipulator. The overall block diagram can be found below in Figure 2.

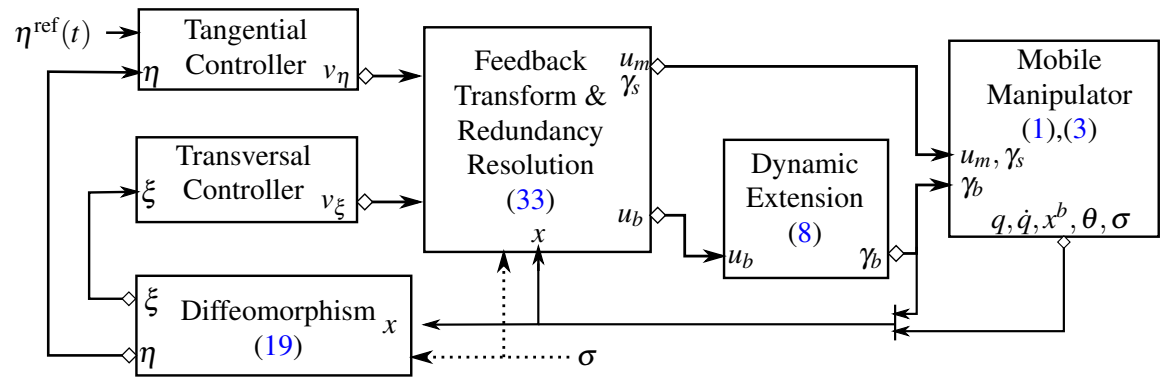

Fig. 2 Path following controller block diagram for mobile manipulator systems.

Dynamic Extension: Dynamic extension amounts to controlling the derivative(s) of the actual control input. Since $\gamma_{b}$ appears too soon by one derivative when computing the derivatives of $y$, we introduce an auxiliary control variable $u_{b} \in \mathbb{R}^{\delta_{b}}$ as follows

$$
\dot{\gamma}_{b}=\rho \gamma_{b}+u_{b}
$$

where $\rho<0$ is a damping coefficient. It is typical with dynamic extension [2] to let $\rho=0$, however we will see in Sect. 4 that setting $\rho=0$ can result in unbounded internal dynamics.

To facilitate the control design procedure, we write the dynamics (1),(3),(8) with output (6) in state-space form. First, let $x_{c}:=\left(q, x^{b}, \theta\right) \in \mathbb{R}^{N} \times \mathbb{R}^{2} \times \mathbb{R}$, $x_{v}:=\left(\dot{q}, \gamma_{b}, \sigma\right) \in \mathbb{R}^{N} \times \mathbb{R}^{\delta_{b}} \times \mathbb{R}^{\delta_{s}}$. Then,

$$
\begin{aligned}
\dot{x}_{c} & =\left[\begin{array}{c}
\dot{q} \\
R(\theta) \Sigma(\sigma) \gamma_{b}
\end{array}\right]=: F_{c}(x) \\
\dot{x}_{v} & =\left[\begin{array}{c}
-M^{-1}(q)(C(q, \dot{q}) \dot{q}+G(q)) \\
\rho \gamma_{b} \\
0_{\delta_{s} \times 1}
\end{array}\right]+\left[\begin{array}{ccc}
M^{-1}(q) A(q) & 0_{N \times \delta_{b}} & 0_{N \times \delta_{s}} \\
0_{\delta_{b} \times N} & I_{\delta_{b} \times \delta_{b}} & 0_{\delta_{s} \times \delta_{s}} \\
0_{\delta_{b} \times N} & 0_{\delta_{b} \times \delta_{b}} & I_{\delta_{s} \times \delta_{s}}
\end{array}\right]\left[\begin{array}{c}
u_{m} \\
u_{b} \\
\gamma_{s}
\end{array}\right] \\
& =: F_{v}(x)+G_{v}(x) u
\end{aligned}
$$


where $x:=\left(x_{c}, x_{v}\right) \in \mathbb{R}^{2 N+3+\delta_{b}+\delta_{s}}$ is the state of the system, $u:=\left(u_{m}, u_{b}, \gamma_{s}\right) \in$ $\mathbb{R}^{N+\delta_{b}+\delta_{s}}$ is the control input to the system, and $y$ is the output. Note that $F_{c}(x)$ and $F_{v}(x)$ are smooth vector fields, $G_{v}(x)$ is smooth and has full rank, and $\frac{\partial H\left(x_{c}\right)}{\partial x_{v}}=0$. The control inputs of the physical system (1),(3) are $u_{m}, \gamma_{b}, \gamma_{s}$, while (8) constitutes the dynamic portion of the controller implemented in the control algorithm.

The state space equations can be compactly written as

$$
\begin{aligned}
& \dot{x}=\left[\begin{array}{c}
\dot{x}_{c} \\
\dot{x}_{v}
\end{array}\right]=\left[\begin{array}{c}
F_{c}(x) \\
F_{v}(x)
\end{array}\right]+\left[\begin{array}{c}
0_{(N+3) \times\left(N+\delta_{b}+\delta_{s}\right)} \\
G_{v}(x)
\end{array}\right] u=: f(x)+g(x) u \\
& y=H\left(x_{c}\right)
\end{aligned}
$$

The mobile manipulator system is redundant in the sense that $\operatorname{dim} u=N+\delta_{b}+$ $\delta_{s} \geq \operatorname{dim} y=p$.

Virtual Outputs: The first virtual output, $\eta_{1}(x)$, is the tangential position of the output $y$ along the desired path. Denote the parameter of curve $\sigma$ that corresponds to the closest point to the output $y$ as $\lambda^{*} \in\left[\lambda_{\min }, \lambda_{\max }\right]$

$$
\lambda^{*}:=\varpi(y):=\underset{\lambda \in\left[\lambda_{\min }, \lambda_{\max }\right]}{\operatorname{arginf}}\|y-\sigma(\lambda)\| .
$$

The minimization for $\lambda^{*}$ is done numerically using a gradient-descent-like algorithm [9]. The first virtual output is the projected, traversed arclength along the curve:

$$
\eta_{1}=\eta_{1}(x):=\left.\int_{\lambda_{\min }}^{\lambda^{*}}\left\|\frac{\mathrm{d} \sigma(\lambda)}{\mathrm{d} \lambda}\right\| \mathrm{d} \lambda\right|_{\lambda^{*}=\varpi \circ H\left(x_{c}\right)}
$$

This integral does not have to be computed for real time implementation if only tangential velocity control is required, as in the case of our experiment (see Sec. 5).

The remaining virtual outputs are selected to represent the cross track error to the path using the remaining FSF normal vectors, known as transversal positions [9]. The generalized Frenet-Serret (FS) vectors are constructed applying the GramSchmidt Orthonormalization process to the vectors $\sigma^{\prime}(\lambda), \sigma^{\prime \prime}(\lambda), \ldots, \sigma^{(p)}(\lambda)$ :

$$
\mathbf{e}_{j}(\lambda):=\frac{\overline{\mathbf{e}}_{j}(\lambda)}{\left\|\overline{\mathbf{e}}_{j}(\lambda)\right\|}
$$

where

$$
\overline{\mathbf{e}}_{j}(\lambda):=\sigma^{(j)}(\lambda)-\sum_{i=1}^{j-1}\left\langle\sigma^{(j)}(\lambda), \mathbf{e}_{i}(\lambda)\right\rangle \mathbf{e}_{i}(\lambda),
$$

for $j \in\{1, \ldots, p\}$. This formulation is well-defined by Assumption 3.1. If FSF are not feasible, other frames may work [8].

The transversal positions can be computed by projecting the error to the path, $y-\sigma\left(\lambda^{*}\right)$, onto each of the FS normal vectors: 


$$
\xi_{1}^{j-1}=\xi_{1}^{j-1}(x):=\left.\left\langle\mathbf{e}_{j}\left(\lambda^{*}\right), H\left(x_{c}\right)-\sigma_{k^{*}}\left(\lambda^{*}\right)\right\rangle\right|_{\lambda^{*}=\varpi \circ H\left(x_{c}\right)}
$$

for $j \in\{2, \ldots, p\}$.

The tangential and transversal positions are illustrated in Figure 3 for $p=3$.

Fig. 3 The tangential and transversal state positions when $p=3$.

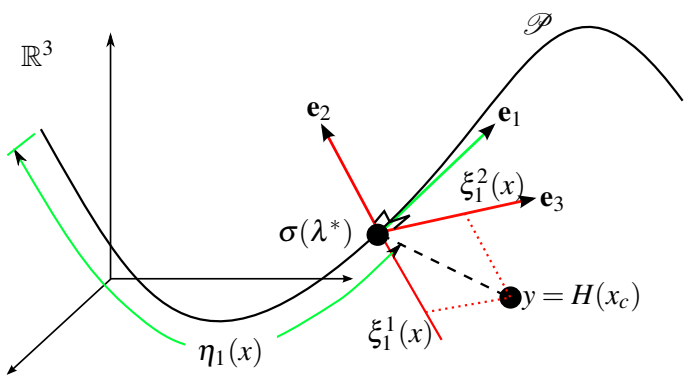

Dynamics and Control: It will be shown that the virtual output $\left(\eta_{1}(x), \xi_{1}^{1}(x), \ldots, \xi_{1}^{p-1}(x)\right) \in$ $\mathbb{R}^{p}$ has a well-defined vector relative degree of $\{2,2, \ldots, 2\}$ for all $x \in \mathscr{U}$, where

$$
\mathscr{U}:=\left\{x \in \mathbb{R}^{2 N+3+\delta_{b}+\delta_{s}} \mid \operatorname{rank}\left(\frac{\partial H}{\partial x_{c}} \frac{\partial F_{c}}{\partial x_{v}} G_{v}(x)\right)=p\right\}
$$

(see (24)) when (14) is solved numerically [9]. We emphasize that (14) is solved for numerically by a local search since if the output $y$ is equidistant to multiple points on the path, then (14) is not well defined and neither is the relative degree. Under these conditions, there exists a local coordinate transformation

$$
\begin{aligned}
T & : \mathscr{U} \rightarrow \mathbb{R}^{2 N+3+\delta_{b}+\delta_{s}} \\
x & \mapsto\left(\eta_{1}(x), L_{f} \eta_{1}(x), \xi_{1}^{1}(x), L_{f} \xi_{1}^{1}(x), \ldots, \xi_{1}^{p-1}(x), L_{f} \xi_{1}^{p-1}(x), \varphi(x)\right) \\
& =\left(\eta_{1}, \eta_{2}, \xi_{1}^{1}, \xi_{2}^{1}, \ldots, \xi_{1}^{p-1}, \xi_{2}^{p-1}, \zeta\right)
\end{aligned}
$$

which is a diffeomorphism onto its image for a suitable choice of the function $\varphi: \mathbb{R}^{2 N+3+\delta_{b}+\delta_{s}} \rightarrow \mathbb{R}^{2 N+3+\delta_{b}+\delta_{s}-2 p}$ [13] (which may limit the domain of $T$ by [13, Proposition 5.1.2], but in practice (see Sect. 5), these functions do not need to be computed unless one wants to visualize the redundant dynamics or study their stability properties). Note that since the virtual output $\eta_{1}$ represents the tangential position of the output $y$ along the path, $\eta_{2}$ represents the tangential velocity of the output along the path. Similarly, since $\xi_{1}^{j}$ represents the transversal positions to the path, $\xi_{2}^{j}$ represents the transversal velocities, $j \in\{1, \ldots, p-1\}$. Note that there will always be an internal state $\zeta$, since by Assumption 2.1, $N+\delta_{b}+\delta_{s} \geq N+\delta_{m} \geq p$ and $\delta_{b}+\delta_{s} \in\{2,3\}$ always implies $2 N+3+\delta_{b}+\delta_{s}-2 p>0$, which is expected as mobile manipulators are highly redundant systems.

The dynamics in the transformed coordinates are 


$$
\begin{array}{ll}
\dot{\eta}_{1}=\eta_{2} & \dot{\xi}_{1}^{j}=\xi_{2}^{j} \\
\dot{\eta}_{2}=L_{f}^{2} \eta_{1}(x)+L_{g} L_{f} \eta_{1}(x) u & \dot{\xi}_{2}^{j}=L_{f}^{2} \xi_{1}^{j}(x)+L_{g} L_{f} \xi_{1}^{j}(x) u \\
\dot{\zeta}=L_{f} \varphi(x)+L_{g} \varphi(x) u=: f_{\zeta}(x, u) &
\end{array}
$$

for $j \in\{1, \ldots, p-1\}$ and $x=T^{-1}(\eta, \xi, \zeta)$. The expressions for the Lie derivatives can be found in [7]. Based on the virtual output construction (Sect. 4), the $\eta$-subsystem represents the dynamics tangent to the path, and the $\xi$-subsystem represents the dynamics transversal to the path. Thus, the dynamics $f_{\zeta}$ represent the dynamics of the system (12) that do not produce any output motion. These are the redundant dynamics to path following. Next we perform standard partial feedback linearization for non-square systems Let

$$
\begin{aligned}
\alpha(x) & :=\left[\begin{array}{ll}
L_{f}^{2} \eta_{1}(x) & L_{f}^{2} \xi_{1}^{1}(x) \ldots L_{f}^{2} \xi_{1}^{p-1}(x)
\end{array}\right]^{\top} \in \mathbb{R}^{p} \\
\beta(x) & :=\left[\begin{array}{c}
L_{g} L_{f} \eta_{1}(x) \\
L_{g} L_{f} \xi_{1}^{1}(x) \\
\vdots \\
L_{g} L_{f} \xi_{1}^{p-1}(x)
\end{array}\right] \in \mathbb{R}^{p \times\left(N+\delta_{b}+\delta_{s}\right)}
\end{aligned}
$$

where the terms in $\beta(x)$ are

$$
\begin{gathered}
L_{g} L_{f} \eta_{1}(x)=\mathbf{e}_{1}\left(\lambda^{*}\right)^{\top} \frac{\partial H\left(x_{c}\right)}{\partial x_{c}} \frac{\partial F_{c}(x)}{\partial x_{v}} G_{v}\left(x_{c}\right) \\
L_{g} L_{f} \xi_{1}^{j-1}(x)=\mathbf{e}_{j}\left(\lambda^{*}\right)^{\top} \frac{\partial H\left(x_{c}\right)}{\partial x_{c}} \frac{\partial F_{c}(x)}{\partial x_{v}} G_{v}(x)+ \\
\left(H\left(x_{c}\right)-\sigma\left(\lambda^{*}\right)\right)^{\top} \mathbf{e}_{j}^{\prime}\left(\lambda^{*}\right) \frac{L_{g} L_{f} \eta_{1}(x)}{\left\|\sigma^{\prime}\left(\lambda^{*}\right)\right\|}
\end{gathered}
$$

for $j \in\{2, \ldots, p\}$ and $\lambda^{*}=\varpi \circ H\left(x_{c}\right)$ [7]. The decoupling matrix $\beta(x)$ has full row rank $p$ in the set $\mathscr{U}$ (see (18)) since each $\mathbf{e}_{i}$ are orthogonal by FSF construction, thus in $U$ the virtual output has a well-defined relative degree [13].

Remark 1. A necessary condition for $\beta(x)$ to have rank $p$ is that each matrix $\frac{\partial H}{\partial x_{c}}, \frac{\partial F_{c}}{\partial x_{v}}$ and $G_{v}(x)$ have rank of at least $p$. The matrix $\frac{\partial H\left(x_{c}\right)}{\partial x_{c}}$ has the form

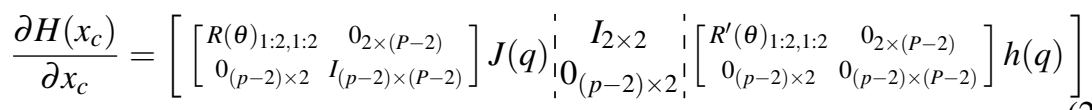

and always has rank of at least 2 due to the ground vehicle (thus is always full rank if $p=2$ ). For $p>2$, it has full rank if $J(q)$ is non-singular.

The matrix $\frac{\partial F_{c}(x)}{\partial x_{v}}$ has the form 


$$
\frac{\partial F_{c}}{\partial x_{v}}=\left[\begin{array}{ccc}
I_{N \times N} & 0_{N \times \delta_{b}} & 0_{N \times \delta_{s}} \\
0_{3 \times N} & R(\theta) \Sigma(\sigma) & R(\theta) \frac{\partial \Sigma(\sigma)}{\partial \sigma}
\end{array}\right]
$$

thus $N+3 \geq \operatorname{rank}\left(\frac{\partial F_{c}}{\partial x_{v}}\right) \geq N+\delta_{b} \geq p$ (by Assumption 2.1) and will depend on the particular ground vehicle. For a unicycle (Sect. 5), for all $x, \operatorname{rank}\left(\frac{\partial F_{c}(x)}{\partial x_{v}}\right)=N+\delta_{b}=$ $N+2$. The matrix $G_{v}(x)$ (see (9)) always has full rank of $N+\delta_{b}+\delta_{s} \geq p$.

Next, introduce an auxiliary input $v:=\left(v_{\eta}, v_{\xi}\right) \in \mathbb{R} \times \mathbb{R}^{p-1}$ such that

$$
v=\beta(x) u+\alpha(x) .
$$

The dynamics (22) then become

$$
\begin{array}{rlrl}
\dot{\zeta}=f_{\zeta}(x, u) & \dot{\eta}_{1}=\eta_{2} & & \dot{\xi}_{1}^{j}=\xi_{2}^{j} \\
\dot{\eta}_{2}=v_{\eta} & & \dot{\xi}_{2}^{j}=v_{\xi^{j}}
\end{array}
$$

where $j \in\{1, \ldots, p-1\}$ and $x=T^{-1}(\eta, \xi, \zeta)$.

In the set $\mathscr{U}$ the $\xi$-subsystem is linear and controllable, and can be stabilized to ensure attractiveness and invariance of the path $\mathscr{P}$ [11], thereby satisfying PF1. The $\eta$-subsystem is also linear and controllable, thus a tangential controller can be designed for $v_{\eta}$ to track a desired tangential position or velocity profile $\eta^{\text {ref }}(t)$, thereby satisfying PF2. Fig. 2 shows a block diagram of the entire control system.

Redundancy Resolution: Once the auxiliary control signal $v \in \mathbb{R}^{p}$ is generated to stabilize the $\eta$ and $\xi$-subsystems for PF1 and PF2, the feedback transformation (29) must be solved to generate the actual control signal $u \in \mathbb{R}^{N+\delta_{b}+\delta_{s}}$. When $N+$ $\delta_{b}+\delta_{s}=p$, there is a unique solution to (29), and one must ensure that the internal dynamics $\dot{\zeta}=f_{\zeta}(x, u)$ remain bounded.

For most mobile manipulator systems (see Sect. 5 for an example), $N+\delta_{b}+\delta_{s}>$ $p$. Thus there is some freedom in the choice of the control input $u$ under the feedback transform (29). This freedom can be used to enforce boundedness of the internal dynamics. We apply the approach from [9] to mobile manipulator systems. Consider the static optimization

$$
\begin{aligned}
& \min _{u}(u-r(x))^{\top} W(u-r(x)) \\
& \text { s.t. } \quad v=\beta(x) u+\alpha(x)
\end{aligned}
$$

This is a static minimization of a quadratic function of $u$ under a linear constraint, for which a closed form solution for $u$ can be found using Lagrange multipliers. One might be tempted to use inequality constraint over $x$ to respect joint limits, but it is unclear how to do so since we are optimizing over the control effort $u$ and $x$ is treated as a parameter. The matrix $W \in \mathbb{R}^{\left(N+\delta_{b}+\delta_{s}\right) \times\left(N+\delta_{b}+\delta_{s}\right)}$ is a positive-definite weighting matrix, and the function $r: \mathbb{R}^{N+3} \rightarrow \mathbb{R}^{N+\delta_{b}+\delta_{s}}$ is used to bias the control input $u$ to achieve desired behaviour in the internal dynamics. For example, if $W$ is the identity matrix and $r(x)$ is the zero function, then we are minimizing control effort while achieving PF1 and PF2. 
In this application, we want to bias $u$ such that the manipulator stays away from joint and actuation limits (PF3). If a joint $q_{i}, i \in\{1, \ldots, N\}$ of the manipulator is at its minimum limit $q_{i}^{\min }$, setting the control effort $u_{i}, i \in\{1, \ldots, N\}$ (i.e., $u_{m}$ ) corresponding to this joint to be the maximum control effort $u_{i}^{\max }$ will likely increase the value of the joint, thereby pushing it away from the negative joint limit. These joint limits can be set artificially to bias the manipulator in a preferred position (so as to avoid singular configurations of $J(q)$, see Remark 1) or set to their true joint limit values. For $u_{i}, i \in\left\{N+1, \ldots, N+\delta_{b}+\delta_{s}\right\}$ (i.e., $u_{b}, \gamma_{s}$ ) we'd like to minimize the control effort so that the mobile base moves as little as possible (however if there are steering limits on $\sigma$, a similar logic used for joint limits on a manipulator can be applied). The corresponding $r$ function to achieve this behaviour in (30) is

$$
\begin{aligned}
& r(x)_{i}:=-\frac{u_{i}^{\max }-u_{i}^{\min }}{q_{i}^{\max }-q_{i}^{\min }}\left(q_{i}-q_{i}^{\min }\right)+u_{i}^{\max }, \quad i \in\{1, \ldots, N\} \\
& r(x)_{i}:=0, \quad i \in\left\{N+1, N+\delta_{b}+\delta_{s}\right\}
\end{aligned}
$$

Using Lagrangian multipliers, the solution to (30) is

$$
u=\beta^{\dagger}(x)(v-\alpha(x))+\left(I_{n \times n}-\beta^{\dagger}(x) \beta(x)\right) r(x)
$$

where $\beta^{\dagger}(x):=W^{-1} \beta(x)^{\top}\left(\beta(x) W^{-1} \beta(x)^{\top}\right)^{-1}$.

In [9, Conjecture 3.5], we showed via examples and experiments on manipulators that a similar control law (33) seems to provide boundedness of the internal dynamics while maintaining joint limits of a manipulator when each degree of freedom has inherent viscous friction. In this paper, for mobile manipulator systems, boundedness of internal dynamics and maintaining joint limits of the manipulator holds when $\rho<0$ in the dynamic extension (8) (PF3). If $\rho=0$, PF1 and PF2 can still be achieved, but the internal dynamics change and at least one of the base position states $\left(x^{b}, \theta\right)$ may become unbounded, see Ex. 1 . The reason is $u_{b}$ controls the mobile base acceleration through (8). So although (31) is chosen such that base acceleration is minimized, ideally keeping $u_{b}=0$, then the base velocities remain at a some constant, not necessarily zero, value, resulting in base states (namely $\theta$ ) that continuously grow. Thus, $\rho<0$ is used to dampen these dynamics. The same doesn't happen for the steering dynamics of $\sigma$ since the steering dynamics are governed by a single integrator. So $\gamma_{s}$ is minimized, ideally 0 when possible, implies the steering position is constant when possible, thus remaining bounded.

Example 1 (Internal Dynamics). Consider the mobile manipulator system of Sec. 5. In this system and in most systems, $u_{i}^{\max }=-u_{i}^{\min }$ for use in (31). The joint limits are set to their true values except for the waist joint $q_{1}$ where $q_{1}^{\min }:=0^{\circ}, q_{1}^{\max }:=20^{\circ}$. Thus the optimal control effort for the waist $r(x)_{1}=0$ when $q_{1}=10^{\circ}$, so that the desired position of the waist is $10^{\circ}$, that is, the manipulator should face to the right of the vehicle (or the vehicle is to stay towards the left of the desired path). The path is shown in Fig. 4, and $\eta_{2}^{\text {ref }}=100 \mathrm{~mm} / \mathrm{sec}$. Two simulation results are included that show PF1 and PF2 are achieved, and there is automatic coordination between the manipulator and the mobile base. When $\rho=0, \mathbf{P F 3}$ is not achieved as the yaw state 
$\theta$ of the mobile base continuously increases and the vehicle orbits the end-effector as the end-effector follows the path.

When $\rho<0$, PF3 is achieved as discussed above. At the very start of the run, the base automatically orients itself by rotating, travels backwards until the end-effector is at its preferred position while the end effector is on the path $\mathscr{P}$, then proceeds forward as it achieves PF2. Note that the initial position of the base could have been placed further away from the path, and the same controller will automatically move the base to a neighbourhood of the path. Further notice the controller automatically speeds up the mobile base when the end-effector is traversing the higher curvature areas of the path in order to maintain the desired constant tangential velocity profile.
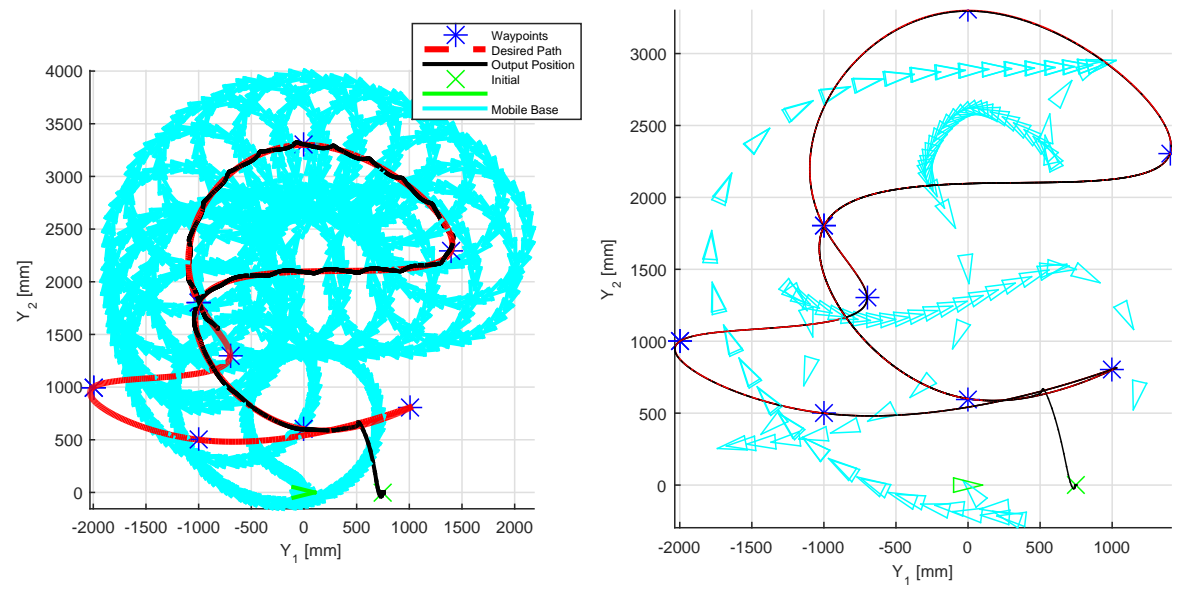

Fig. 4 Example 1: Simulation of $\rho=0$ (left) and $\rho=-10$ (right). When $\rho=0$, the mobile base orbits the output position $y=H\left(x_{c}(t)\right)$ such that it maintains the preferred position and is on the desired path $\mathscr{P}$. Each snapshot of the mobile base is taken every 1.5 seconds.

Remark 2. The weighting matrix $W$ plays an important role to ensure the joint limits are satisfied. If the associated weights for the manipulator joint actuation are too low, the controller may not move the mobile base at all before the manipulator reaches a singular configuration. If the weights are high enough, then the controller will increase control effort to the base so that the base moves while the manipulator satisfies its joint limits.

\section{Experiment}

System Model: The mobile manipulator system is a platform designed by Clearpath Robotics (see Fig. 5). The manipulator is a 4-degree-of-freedom system. The com- 
plete modelling procedure can be found in [8], yields the dynamics of the manipulator in form (1) with $N=4$ and $u_{m}$ representing the vector of control input voltages to the motors. The mobile platform runs a low level control loop that controls the vehicle's heading rate and tangential velocity. The vehicle has no steering wheels. Thus, for this system $\delta_{s}=0$ and $\delta_{b}=2$ and the general kinematic model (3) reduces to the standard unicycle equations [5]

$$
\begin{array}{r}
\dot{x}_{1}^{b}=\gamma_{b_{1}} \cos (\theta) \\
\dot{x}_{2}^{b}=\gamma_{b_{1}} \sin (\theta) \\
\dot{\theta}=\gamma_{b_{2}}
\end{array}
$$

The output space is taken to be the 3-dimensional Euclidian space, that is $p=3$.

Path Following Controller: The desired path is generated by spline-interpolating 3-dimensional waypoints using quintic polynomials [6]. The control design approach then follows from Sec. 4. For this mobile manipulator system, significant modelling uncertainties arise due to inaccurate manipulator modelling [8] and the assumption of a perfect kinematic model of the mobile base, as well as ignoring the coupling dynamics between the two. This results in imperfect cancellation of the actual system dynamics using (23),(24). The Lyapunov redesign based robust controller in [8, Eq. (27)] is used to overcome the modelling uncertainties, thereby achieving PF1.

The $\eta$-subsystem is also linear and controllable, thus a tangential controller can be designed for $v_{\eta}$ to track some desired tangential position or velocity profile $\eta^{\text {ref }}(t)$. Our goal is to track a desired constant velocity profile $\eta_{2}^{\text {ref }}$. This can be done using a PI controller [8, Eq. (24)] where the integral action is used for robustness, thereby achieving PF2. Fig. 2 has the complete block diagram.

The manipulator has a Labview Real-Time Module ${ }^{\circledR}$ which is used to read the linear actuator distances using optical encoders and to control the motor PWM amplifiers. The encoder readings are converted to joint angles $q$ and numerically differentiated to approximate $\dot{q}$. This module communicates with ROS via the Rosbridge package. The Husky is a ROS-enabled robot which takes in $\gamma_{b}$ to control the robot, and gives out $\left(x^{b}, \theta\right)$ data based on a sensor fusion of wheel odometry and an onboard IMU. The path following controller is implemented using MATLAB Robotics System Toolbox.

Results: The joint limits in (31) are set so that in Fig. 6, the waist of the manipulator prefers $90^{\circ}$ (the manipulator is ahead of the vehicle), and again in another run in Fig. 10, so that the waist prefers $160^{\circ}$ (the manipulator is to the left of the vehicle). In all cases, $\eta_{2}^{\text {ref }}=100 \mathrm{~mm}$.

As shown in the 3D and 2D plots, the proposed path following controller successfully satisfies the goals PF1 and PF3. The output $y$ automatically goes towards the closest point on the path $\mathscr{P}$ due to the coordinate transformation employed. A key advantage of the proposed approach is the automatic coordination of the manipulator and mobile base. Based on the preferred position of the manipulator via (31), the mobile base automatically orients itself. In the second lap of the path (indicated in magenta), the mobile base actually moves backwards until $x^{b} \approx(-1000,500) \mathrm{mm}$, 
at which point the base turns and moves forward in order to keep the manipulator at its preferred position and to traverse the path at the desired rate $\eta_{2}^{\text {ref }}$, without any explicit trajectory planning and tracking for the mobile base.

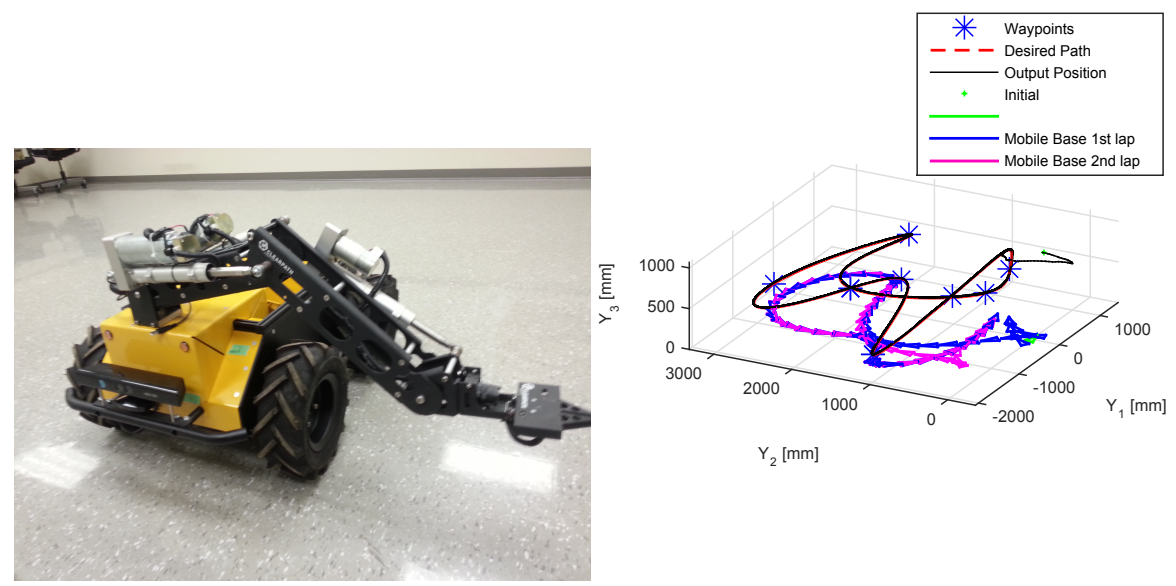

Fig. 5 Clearpath manipulator mounted on a Fig. 6 Experiment 3D response - preferred $q_{1}=$ Clearpath A200 mobile platform. $90^{\circ}$.

We see that in the state trajectory (Fig. 8), the manipulator positions $q$ quickly converge to their preferred positions when possible, in particular, the waist $q_{1}$ approaches $90^{\circ}$. The virtual output trajectories can be found in Fig. 9 and show that the system is traversing the path at a constant (desired) rate (PF2), while the transversal errors quickly approach 0 resulting in convergence to the desired path $\mathscr{P}(\mathbf{P F 1})$. At steady state, the cross-track errors are less than $\approx 15 \mathrm{~mm}$.

When the preferred waist position is adjusted, the mobile base automatically takes another route for the same desired path $\mathscr{P}$. In Fig. 10, it can be seen that the mobile base tries to stay to the right of the path in order to respect the artificial preferred position of the waist of the manipulator. Note that at the high curvature areas, the mobile base speeds up significantly (apparent by the decreased density of the mobile base snapshots) in order to respect PF2.

\section{Conclusions and Future Work}

This paper proposes a unified path following controller for mobile manipulator systems. The controller automatically moves the mobile base and the manipulator such that the end-effector traverses a path in the output space towards a given desired position or with a given velocity profile. There is no explicit trajectory required for the mobile base or the end-effector to follow. The desired path is rendered invariant and attractive, and the redundancy resolution scheme employed allows for the ma- 


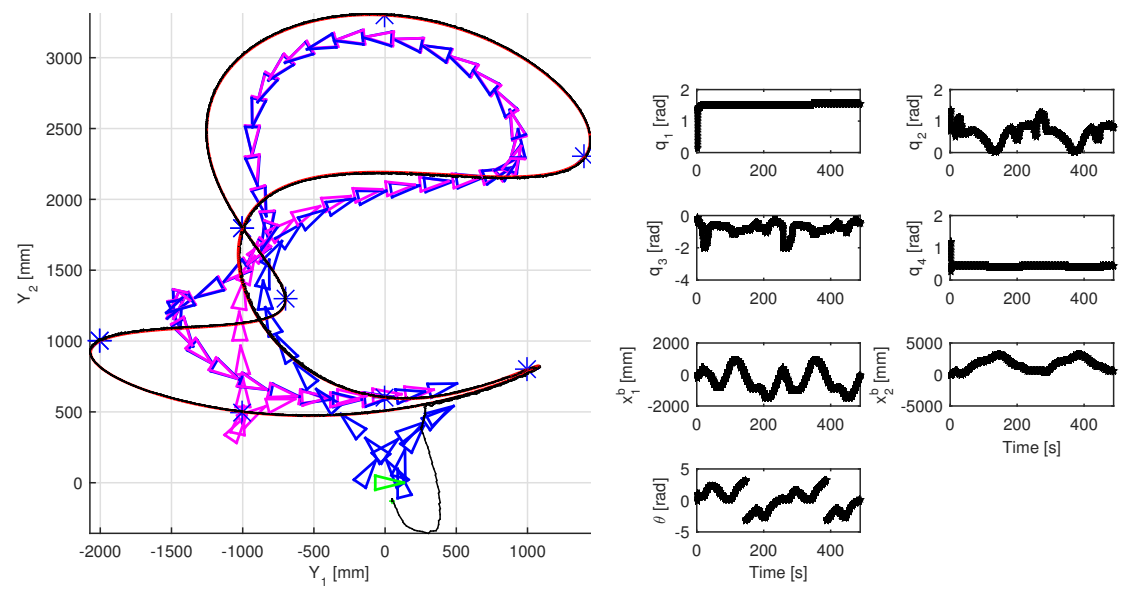

Fig. 7 Experiment response, top view - preferred $q_{1}=90^{\circ}$. Each snapshot of the mobile base is Fig. 8 Preferred $q_{1}=90^{\circ}$. State position $x_{c}$ trataken every 4 seconds. jectories.
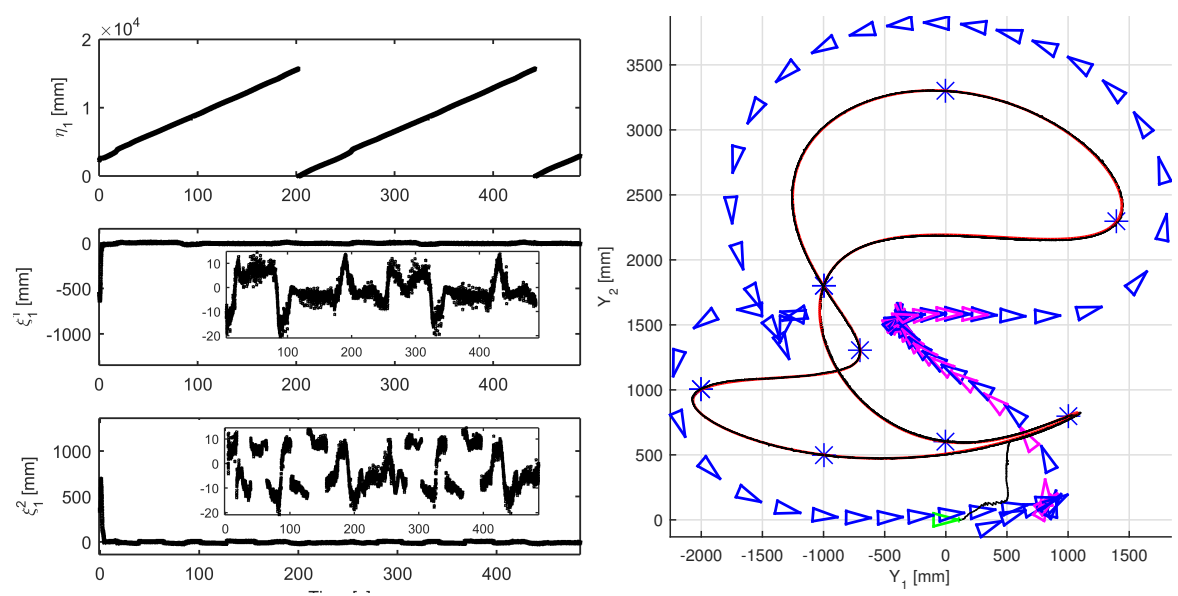

Fig. 9 Preferred $q_{1}=90^{\circ}$. Virtual output trajec- Fig. 10 Experiment response, top view - pretories. Notice from $\eta_{1}$ just over 2 laps are com-ferred $q_{1}=160^{\circ}$. Each snapshot of the mobile pleted. base is taken every 4 seconds.

nipulator to stay away from joint limits. Dynamic extension with damping was used so that the virtual output employed for path following has a full relative degree and to ensure boundedness of the internal dynamics.

Dynamically changing the $r$ function in (31) for real-time obstacle avoidance is a direction for future work. Analyzing when the virtual output constructed in the mobile manipulator path following controller loses full relative degree (that is when (24) loses rank) is another direction for future work. This will help determine which configurations of the mobile manipulator with respect to the path should be avoided. 


\section{References}

[1] Aguiar A, Hespanha J, Kokotovic P (2005) Path-following for nonminimum phase systems removes performance limitations. IEEE Transactions on Automatic Control 50(2):234-239

[2] Akhtar A, Nielsen C, Waslander S (2015) Path following using dynamic transverse feedback linearization for car-like robots. IEEE Transactions on Robotics 31(2):269-279

[3] Cameron J, MacKenzie D, Ward K, Arkin R, Book W (1993) Reactive control for mobile manipulation. In: IEEE ICRA, pp 228-235 vol.3

[4] Chung JH, Velinsky S (1998) Modeling and control of a mobile manipulator. Robotica 16(06):607-613

[5] De Wit CC, Bastin G, Siciliano B (1996) Theory of robot control. Springer-Verlag New York, Inc.

[6] Erkorkmaz K, Altintas Y (2001) High speed CNC system design. Part I: Jerk limited trajectory generation and quintic spline interpolation. International Journal of Machine Tools and Manufacture 41(9): 1323 - 1345

[7] Gill R (2015) Robust spline path following for redundant mechanical systems. Master's thesis, University of Waterloo

[8] Gill R, D Kulić D, Nielsen C (2013) Robust path following for robot manipulators. In: IEEE IROS, pp 3412-3418

[9] Gill R, Kulić D, Nielsen C (2015) Spline path following for redundant mechanical systems. ArXiv CoRR Robotics abs/1504.06917

[10] Hauser J, Hindman R (1995) Maneuver regulation from trajectory tracking: Feedback linearizable systems. In: Proc. IFAC Symp. Nonlinear Control Systems Design, pp 595-600

[11] Hladio A, Nielsen C, Wang D (2013) Path following for a class of mechanical systems. IEEE Transactions on Control Systems Technology 21(6):2380-2390

[12] Inoue F, Muralami T, Ihnishi K (2001) A motion control of mobile manipulator with external force. IEEE/ASME Transactions on Mechatronics 6(2):137-142

[13] Isidori A (1995) Nonlinear control systems, 3rd edn. Springer-Verlag

[14] Khatib O (1987) A unified approach for motion and force control of robot manipulators: The operational space formulation. IEEE Journal of Robotics and Automation 3(1):43-53

[15] Khatib O (1999) Mobile manipulation: The robotic assistant. Robotics and Autonomous Systems 26(23): $175-183$

[16] Kreyszig E (1991) Differential Geometry. Differential Geometry, Dover Publications

[17] Lapierre L, Soetanto D (2007) Nonlinear path-following control of an auv. Ocean engineering 34(11):1734-1744

[18] Nagatani K, Yuta S (1996) Designing strategy and implementation of mobile manipulator control system for opening door. In: IEEE ICRA, vol 3, pp 2828-2834 vol.3

[19] Nielsen C, Maggiore M (2006) Output stabilization and maneuver regulation: A geometric approach. Systems \& Control letters 55(5):418-427

[20] Pennock G, Kassner D (1993) The workspace of a general geometry planar three-degree-offreedom platform-type manipulator. Journal of Mechanical Design 115(2):269-276

[21] Peterson L, Austin D, Kragic D (2000) High-level control of a mobile manipulator for door opening. In: IEEE/RSJ International Conference on Intelligent Robots and Systems, 2000, vol 3, pp 2333-2338 vol.3, DOI 10.1109/IROS.2000.895316

[22] Seraji H (1998) A unified approach to motion control of mobile manipulators. The International Journal of Robotics Research 17(2):107-118

[23] Spong M, Hutchinson S, Vidyasagar M (2006) Robot Modeling and Control. John Wiley \& Sons

[24] de Wit CC, Siciliano B, Bastin B (1997) Theory of Robot Control. Springer, New York

[25] Xu Y, Brown J HB, Friedman M, Kanade T (1994) Control system of the self-mobile space manipulator. IEEE Transactions on Control Systems Technology 2(3):207-219

[26] Yamamoto Y, Yun X (1992) Coordinating locomotion and manipulation of a mobile manipulator. In: IEEE Conf. on Decision and Control, pp 2643-2648 